\title{
Water as a promoting media for 1,3-dipolar cycloaddition of phosphorylated azides to internal alkynes
}

\author{
Oleg I. Artyushin, Ekaterina V. Matveeva, Ivan S. Bushmarinov, and Irina L. Odinets* \\ A.N. Nesmeyanov Institute of Organoelement Compounds, Russian Academy of Sciences, \\ Vavilova str., 28, 119991 Moscow GSP-1, Russian Federation
}

E-mail: odinets@ineos.ac.ru

The Commemorative Issue in Honor of Prof. Pawel Kafarski

\begin{abstract}
The 1,3-cycloaddition of $\omega$-phosphoryl azides to activated internal alkynes such as dimethyl acetylenedicarboxylate and tetramethyl acetylenediphosphonate as well sodium azide to tetramethyl acetylenediphosphonate readily proceeds in water without any co-solvent or additive to afford the corresponding phosphorylated 1,2,3-triazoles in excellent yields. The ester group in carbalkoxy function of these compounds can be easily converted to carbamido group via the reaction with amines in $\mathrm{MeOH}$ thereby expanding the range of potentially biologically active heterocyclic aminophosphonates of such type.
\end{abstract}

Keywords: Green chemistry, water reaction, cycloaddition, phosphorylated 1,2,3-triazoles

\section{Introduction}

Among the reactions used nowadays for design of specifically acting low molecular weight molecules for modern drug discovery, so called "click" methodology ${ }^{1}$ allowing quick and easy generation of large libraries of compounds became more and more used. The main principles of the above methodology comprise modular and wide in scope reactions giving very high yields and great diversity of products. These reactions of readily available starting substrates should proceed either in the absence of solvent or in a solvent that is benign (preferably water) to favor a reaction with one single reaction product. The most popular among the click reactions are cycloadditions of unsaturated species, especially 1,3-dipolar Huisgen cycloadditions of azides and terminal alkynes to give the corresponding triazoles. ${ }^{2}$ The discovery of catalytic properties of copper(I) which allows high rate and control of regioselectivity of this cycloaddition in the case of terminal alkynes (therefore referred also as $\mathrm{Cu}$-Alkyne-Azide Cycloaddition or $\mathrm{CuAAC}$ reaction) to give 1,4-disubstituted 1,2,3-triazoles stimulated the investigations in this field over 
the last years. ${ }^{3}$ Even despite the fact that 1,2,3-triazole structural moiety does not occur in nature, a wide range of compounds containing this functionality exhibit diverse biological activities such as triazole-linked glycoconjugates ${ }^{4}$ and glycopeptides ${ }^{5}, 1,2,3$-triazole modified nucleic acids, ${ }^{6}$ nucleoside analogues, ${ }^{7}$ and a range of anti-HIV ${ }^{8}$, anti-epileptic ${ }^{9}$ or antimicrobial ${ }^{10}$ substances. $^{2}$

Taking into account that a wide range of organophosphorus compounds exhibit different types of bioactivity, ${ }^{11}$ recently we have elaborated the effective syntheses of phosphorylated terminal alkynes ${ }^{12,13}$ and azides, ${ }^{14}$ which were involved in $\mathrm{Cu}(\mathrm{I})$ cycloadditions to afford a range of potential drug-candidates such as 1,2,3-triazole-substituted methylenebisphosphonates (NBPs ${ }^{12}$ and the related phosphonocarboxylates (N-PCs), ${ }^{13}$ including those bearing sugar, adamantane, and azidothimidine biogenic moieties, as well as 4-(1H-1,2,3-triazol-1yl)phosphonates having amino acid residues ${ }^{14}$ and perfluorinated groups. ${ }^{15}$

In continuation of this study, in this paper we report on the reactivity of phosphorylated azides towards internal alkynes providing, in water as a sole reaction medium, the facile synthesis of the new polyfunctionalized phosphorus-substituted 1,2,3-triazoles.

\section{Results and Discussion}

Electron-withdrawing groups on acetylenes are well-known to increase the cycloaddition reaction rate, however, under classical conditions the reactions with azides proceed not too fast even for activated internal alkynes. For example, 1,3-dipolar cycloaddition of organic azides to 1-trimethylsilyl carbalkoxy-acetylenes was completed over $14 \mathrm{~h}$ in refluxing toluene, ${ }^{16}$ in the case of dimethyl acetylenedicarboxylate (DMAD), the reaction required prolonged heating of the components (e.g., $90{ }^{\circ} \mathrm{C}$ for $6 \mathrm{~h}^{17}$ or $80{ }^{\circ} \mathrm{C}, 19 \mathrm{~h}^{18}$ ) in the absence of the solvent. The cycloadditions of internal alkynes can be promoted by microwave irradiation ${ }^{19}$ or application of copper(I) or ruthenium(II) catalysts. ${ }^{20}$ Besides MW or application of catalysts, the reaction rate may be optimized by addition of water and application of water-alcohol media (usually $\left.\mathrm{tBuOH}: \mathrm{H}_{2} \mathrm{O} / 4: 1\right)$ as often used for $\mathrm{CuAAC}$ reactions.

Despite the strong acceleration effect of water for many organic reactions, ${ }^{21}$ where low miscibility or solubility of organic compounds is not detrimental and in some cases facilitates the isolation of products, ${ }^{22}$ not too many examples of alkyne-azide cycloadditions have been performed in pure water as a reaction medium ${ }^{21 \mathrm{e}}$ using more often terminal $^{23}$ than internal ${ }^{23 a, 24}$ alkynes. For alkynes of both types the reactions were carried out in the presence of different catalysts and only a few publications reported on cycloaddition without any additives (in three cases of four DMAD was used as an alkyne). ${ }^{1 \mathrm{~b}, 23 a, 25}$ Note that in these cases the additional functionalities in the starting azide did not tolerate the aqueous reactions due to partial hydrolysis. ${ }^{26}$ Apparently, the warnings relating to the possible instability of organophosphorus compounds in water resulted in its limited application in organophosphorus synthesis (the known examples comprise the Wittig, Kabachnik-Fields, aza-Michael reactions and synthesis of 
functionalized stabilized phosphorus ylides via the three-component reaction of triphenylphosphine, dialkyl acetylenedicarboxylates and various nucleophiles). ${ }^{27}$

We have found that cycloaddition of $\omega$-phosphorylalkyl azides 1a,b to DMAD 2a proceeded rapidly in water as a sole solvent without a catalyst to afford 4-phosphorylalkyl-1,2,3-triazoles $\mathbf{3 a}, \mathbf{b}$ in excellent yields and did not affect the ester groups at the phosphorus atom. The reactions were completed in $36 \mathrm{~h}$ at room temperature or $1.5-2 \mathrm{~h}$ at $60{ }^{\circ} \mathrm{C}$. Note that phosphorylated azides $\mathbf{1 a}, \mathbf{b}$ and final products $\mathbf{3 a}, \mathbf{b}$ are soluble in water in contrast to DMAD and formation of homogeneous mixture visually indicates the completion of the reaction. Similarly, phosphorylated azides react readily with tetramethoxy acetylenediphosphonate $\mathbf{2} \mathbf{b}$. As the latter is soluble in water, in this case the homogeneous reaction was completed over shorter period of time. The products 3a-c were isolated by extraction with DCM followed by chromatography via short plug of silica gel.

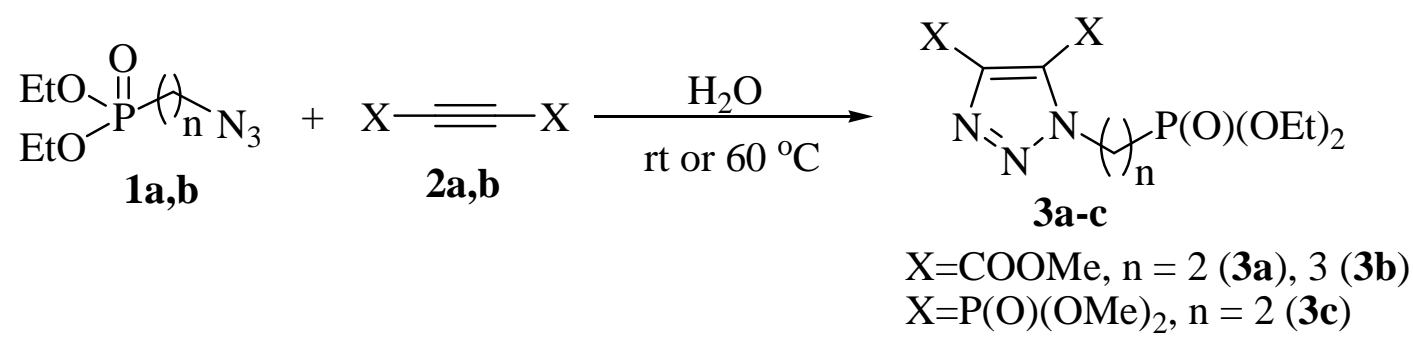

Scheme 1. Reaction of phosphorylated azides with internal alkynes in water.

Application of $\mathrm{Cu}(\mathrm{I})$ salts $(\mathrm{CuI}$ or $\mathrm{CuBr})$ as catalysts does not influence significantly the reaction rate but required additional work-up to remove the copper impurities from the final products. Moreover, in $\mathrm{tBuOH}: \mathrm{H}_{2} \mathrm{O}$ mixed solvent the reaction in which $\mathrm{Cu}(\mathrm{I})$ catalyst was generated in situ from $\mathrm{CuSO}_{4}$ and sodium ascorbate, was accompanied $\left(50{ }^{\circ} \mathrm{C}\right)$ by partial DMAD polymerization and required application of DMAD excess for quantitative conversion of the starting azide.

Addition of sodium azide to acetylenediphosphonate $\mathbf{2 b}$ proceeded in water even more faster giving, in $0.5 \mathrm{~h}$ at room temperature, the corresponding sodium salt 4 in quantitative yield which may be easily isolated by simple removal of water.

In contrast, addition of sodium azide to $\mathrm{DMAD}^{28}$ or to the related bis(diphenyl(thio)phosphoryl)acetylene ${ }^{29}$ performed in $\mathrm{MeOH}$ and $\mathrm{DMF}$ required additional work-up procedures. The reaction in water is exothermic and reasonable dilution of the reaction mixture (see experimental) and maintenance of the temperature below $20{ }^{\circ} \mathrm{C}$ are needed to avoid formation of side products. Further acidification of reaction mixture is accompanied by prototropic schift to yield the $2 \mathrm{H}$-1,2,3-triazole-diphosphonate $\mathbf{5}$ which structure was unambiguously confirmed by X-ray analysis of a single crystal. 


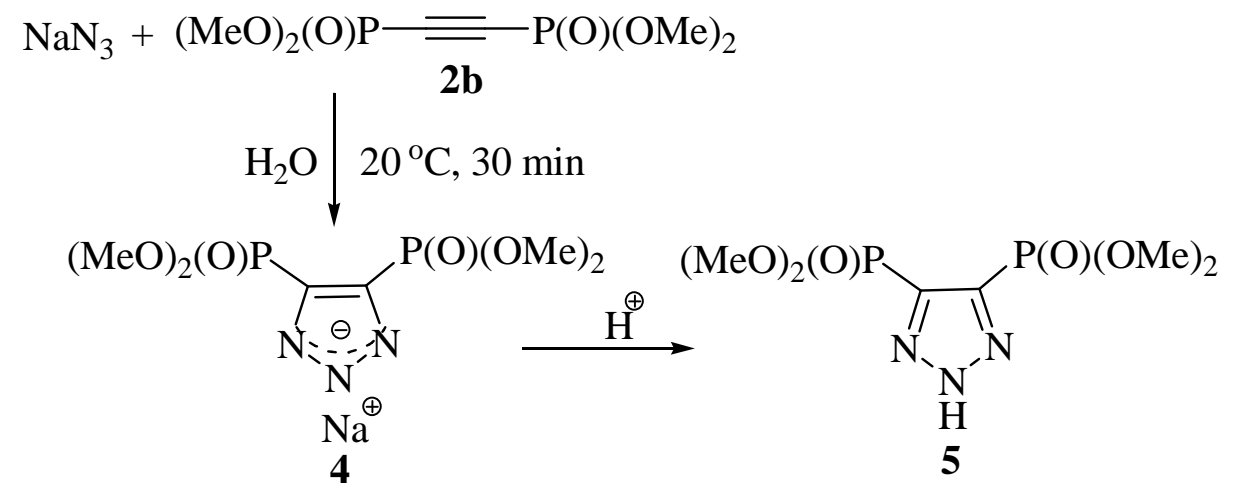

Scheme 2. Synthesis of the tetramethyl $2 H$-1,2,3-triazole-diphosphonate 5 in water.

In crystalline form the molecule of $\mathbf{5}$ (Figure 1) occupied a special position around a $\mathrm{C}_{2}$ axis passing through the N1 atom. Similar to 4,5-bis(diphenylthiophosphinoyl)-1,2,3-triazole, ${ }^{29 \mathrm{~b}}$ the molecule of 5 was present in a $2 \mathrm{H}$ tautomeric form. The C1-P1 bond $(1.7859(18) \AA$ ) was by 0.04 $\AA$ shorter than that in the above mentioned analog with diphenylthiophosphoryl groups 4,5 (1.826 ̊), indicating less steric crowding.

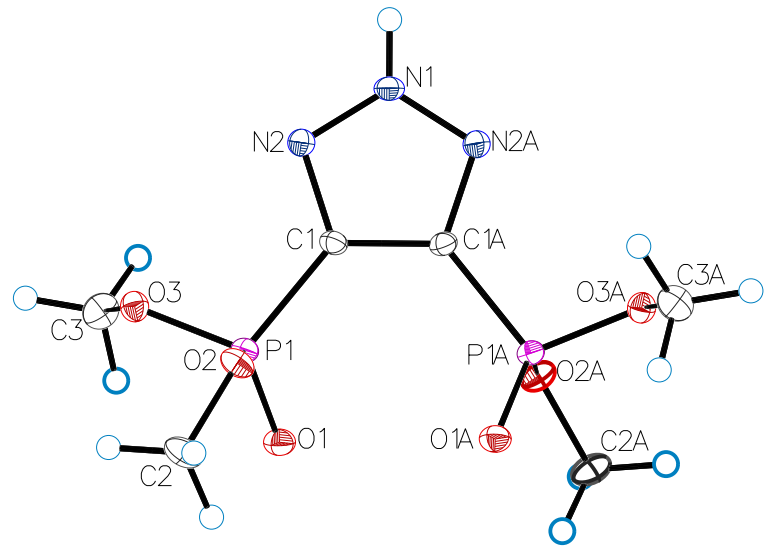

Figure 1. The general view of $\mathbf{5}$ in crystal. Atoms are represented by thermal ellipsoids. Labels with suffix " $\mathrm{A}$ " denote atoms generated by an $(0.5-\mathrm{x}, 0.5-\mathrm{y}, \mathrm{z})$ symmetry operation.

The crystal of 5 consists of infinite chains of bifurcate $\mathrm{N}-\mathrm{H} \ldots \mathrm{O}$ hydrogen bonds $(\mathrm{N} \ldots \mathrm{O}$ $2.877(2) \AA ̊ ., N-H . . O ~ 135.5(6)^{\circ}$, O...H... 88.9(13) $)^{\circ}$ ) (Figure 2) along the crystallographic axis $c$ formed by the phosphoryl oxygen atoms and the hydrogen at the nitrogen atom in the 2-position of the heterocycle. 


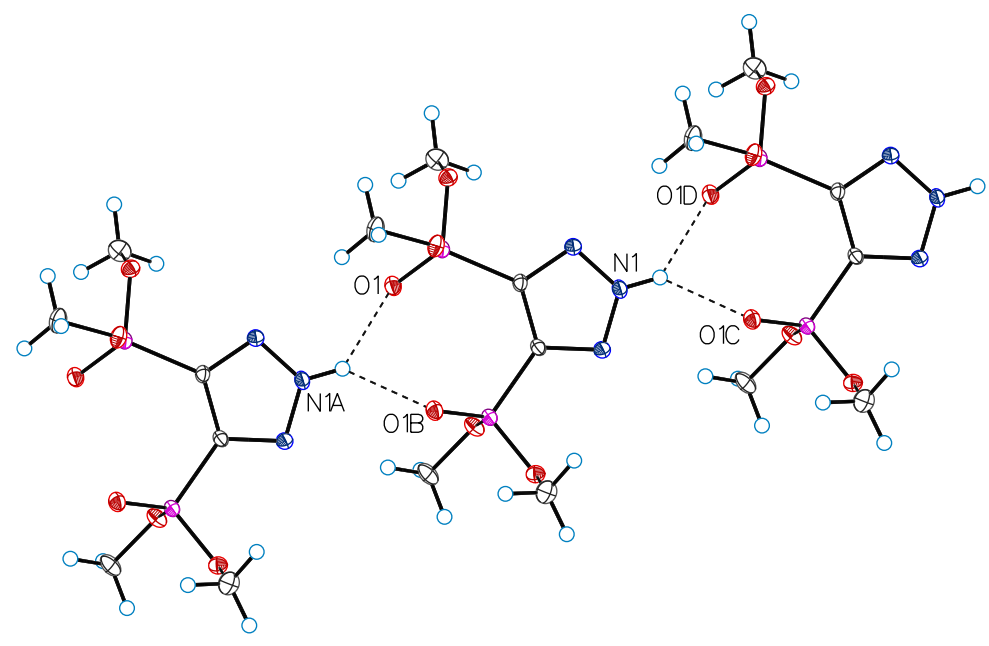

Figure 2. The chain of hydrogen bonds in crystal of 5. Atoms are represented by thermal ellipsoids. The symmetry operations used for generation of $\mathrm{N} 1 \mathrm{~A}, \mathrm{O} 1 \mathrm{~B}, \mathrm{O} 1 \mathrm{C}$ and $\mathrm{O} 1 \mathrm{D}$ atoms are $(+\mathrm{x},+\mathrm{y},-1+\mathrm{z}),(0.5-\mathrm{x}, 0.5-\mathrm{y},+\mathrm{z}),(0.5-\mathrm{x}, 0.5-\mathrm{y}, 1+\mathrm{z})$, and $(+\mathrm{x},+\mathrm{y}, 1+\mathrm{z})$, respectively.

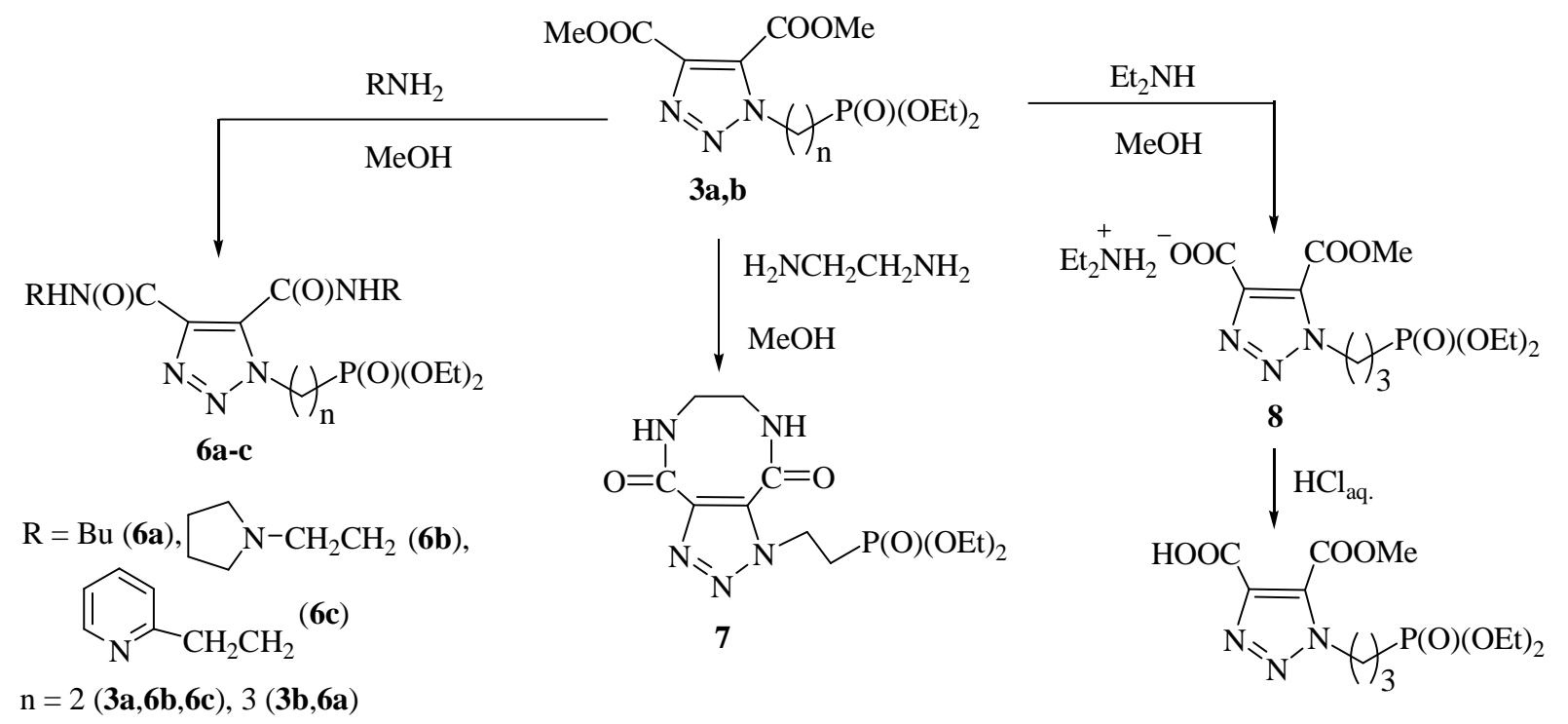

Scheme 3. Amidation of 1,2,3-triazole-4,5-dicarboxylates 3a,b with primary amines.

In addition, to expand the range of such potentially biologically active substances, triazoles 3a,b bearing methoxycarbonyl groups were transformed into the corresponding amides $\mathbf{6 a - c}$ via the reaction with primary amines in $\mathrm{MeOH}$. Reaction proceeded at room temperature and completed over 12,14 and 24 hours in the case of pyrrolidinethylamine, 2-pyridinethylamine and butylamine, respectively. To obtain bicyclic product 7 the reaction with ethylenediamine was performed in very dilute solution over 7 days. It should be noted that the attempt to synthesize 
compound 6a via one-pot three-component reaction of DMAD, phosphorylated azide and butylamine in water was unsuccessful. Moreover, the treatment of the aqueos reaction mixture containing preformed triazole $\mathbf{3 b}$ with butylamine was accompanied by partial hydrolysis of carbomethoxy groups to afford the target bisamide $\mathbf{6 a}$ and the corresponding bis(butylammonium) salt in approximately 1:1 ratio. Application of secondary amines in this reaction resulted in considerable decrease of the reaction rate and change of the reaction course. Thus, the reaction of $\mathbf{3 b}$ and $\mathrm{Et}_{2} \mathrm{NH}$ in a month led to diethylammonium salt $\mathbf{8}$ rather than in the desired amide.

The final 1,2,3-triazoles 3a-c were isolated as yellowish oils in $87-95 \%$ yields by extraction with DCM followed by flash-chromatography on silica gel. The amides 6a-c, 7 were isolated with high purity after removing of volatiles from the reaction mixtures followed by drying in vacuo. In the ${ }^{31} \mathrm{P}$ NMR spectra 1,2,3-triazole-4,5-dicarboxylates 3a,b demonstrated singlets at ca. 25 and $30 \mathrm{ppm}$, respectively, which were slightly upfield shifted comparing with the signals for the starting azides. Vice versa, the signals of the amide derivatives 6a-c and $\mathbf{7}$ are slightly downfield shifted relative the resonances of their carbomethoxy-substituted precursors. Nevertheless, the disposition of the signals has the general pattern: in the case of 1,2,3-triazoleethylphosphonates 3a, 6b,c, and 7 the signals were observed at ca 25-26 ppm and at ca $30 \mathrm{ppm}$ for the related propylphosphonates $\mathbf{3 b}, \mathbf{6 a}$. The signals of phosphorus atoms in the molecule of trisphosphorylated 1,2,3-triazole $\mathbf{3 c}$ were observed at ca. 4 and $7.5 \mathrm{ppm}$ for the phosphonate groups attached to the triazole ring and at $\sim 25 \mathrm{ppm}$ for the phosphonate moiety attached to the ring through an alkylene chain. Taking into account that the signal of phosphonate groups in the starting substrate $\mathbf{2 b}$ is observed at $c a$. $-8 \mathrm{ppm}$, the singlets at 4 and $7.5 \mathrm{pm}$ were assigned to phosphonate groups in 5- and 4-positions of the heterocycle in $3 \mathbf{c}$. The general pattern of the ${ }^{1} \mathrm{H}$ and ${ }^{13} \mathrm{C}$ NMR spectra fit well to the depicted structures. In the IR spectra of the compounds the characteristic bands of phophoryl and carbonyl groups were observed at 1266-1280 and 1735 $\mathrm{cm}^{-1}$ in the case of esters 3a-c and at 1245-1253 and $\sim 1676 \mathrm{~cm}^{-1}$ for the amide derivatives 6a-c,7.

\section{Conclusions}

The phosphorylated azides react with symmetric internal alkynes, both phosphorylated and nonphosphorylated, and in this case the highest reaction rate was observed in water without any cosolvent or additive. This approach is especially advantageous from green chemistry point of view. Furthermore, the alkoxycarbonyl substituted derivatives could be further transformed to corresponding amides thereby expanding the range of potentially biologically active heterocyclic aminophosphonates of such type. 


\section{Experimental Section}

General. NMR spectra were recorded with a Bruker AMX-300 spectrometer using residual proton signals of deuterated solvent as an internal standard $\left({ }^{1} \mathrm{H},{ }^{13} \mathrm{C}\right)$ and $\mathrm{H}_{3} \mathrm{PO}_{4}\left({ }^{31} \mathrm{P}\right)$ as an external standard. The ${ }^{13} \mathrm{C}$ NMR spectra were registered using the JMODECHO mode; the signals for the $\mathrm{C}$ atom bearing odd and even numbers of protons have opposite polarities. IR spectra were recorded on a Fourier-spectrometer "Magna-IR750" (Nicolet), resolution $2 \mathrm{~cm}^{-1}$, 128 scans. Melting points were determined with MPA 120 EZ-Melt Automated Melting Point Apparatus and were uncorrected. Dimethyl acetylenedicarboxylate, amines and sodium azide were purchased from Aldrich and used without further purification.

\section{Crystal structure determination of (5)}

Single crystals of $\mathrm{C}_{6} \mathrm{H}_{13} \mathrm{~N}_{3} \mathrm{O}_{6} \mathrm{P}_{2}(\mathbf{5})$ were grown from $\mathrm{CH}_{2} \mathrm{Cl}_{2}$. A suitable crystal was selected and diffraction data were collected on a Bruker APEX-II CCD diffractometer at 100(2) K with Mo $\mathrm{K} \alpha$ radiation by $\omega / 2 \theta$ scan mode. Using Olex $2^{30}$, the structure was solved with the $\mathrm{XS}^{31}$ structure solution program using direct methods and refined with the $\mathrm{XL}^{31}$ refinement package.

Crystal/refinement data for (5). $\mathrm{C}_{6} \mathrm{H}_{13} \mathrm{~N}_{3} \mathrm{O}_{6} \mathrm{P}_{2}, M=285.13$, orthorhombic, $a=13.777(2) \AA, b=$ 23.966(3) $\AA, c=6.9393(11) \AA, U=2291.2(6) \AA^{3}, T=100$ (2), space group Fdd2 (no. 43), $Z=8$, $\mu(\mathrm{MoK} \alpha)=0.402,4249$ reflections measured, 1637 unique $\left(R_{\text {int }}=0.0292\right)$ which were used in all calculations. The final $w R\left(F_{2}\right)$ was 0.0653 (all data). CCDC 846876 contains the supplementary crystallographic data for this paper. These data can be obtained free of charge from The Cambridge Crystallographic Data Centre via www.ccdc.cam.ac.uk/data_request/cif.

\section{1,2,3-Triazole-4,5-dicarboxylates (3a,b). General procedure}

A mixture of azidophosphonate $\mathbf{1}(2 \mathrm{mmol})$ and dimethyl acetylenedicarboxilate $\mathbf{2 a}(2 \mathrm{mmol})$ in water $(9 \mathrm{ml})$ was stirred at $55-65^{\circ} \mathrm{C}$ for $2 \mathrm{~h}$. After cooling to ambient temperature the mixture was extracted with methylene chloride ( $3 \times 15 \mathrm{ml})$, dried over $\mathrm{Na}_{2} \mathrm{SO}_{4}$, filtered and concentrated. The residue was purified by column chromatography on silica gel (acetone-hexane=1:1).

Dimethyl 1-[3-(diethoxyphosphoryl)ethyl]-1H-1,2,3-triazole-4,5-dicarboxylate (3a). Yellow oil, yield 87\%; IR (thin layer, $\left.v_{\max }, \mathrm{cm}^{-1}\right)$ : 1026, $1061(\mathrm{P}-\mathrm{O}-\mathrm{C}), 1267(\mathrm{P}=\mathrm{O}), 1735(\mathrm{C}=\mathrm{O}) .{ }^{31} \mathrm{P}$ NMR (121.5 MHz, $\left.\mathrm{CDCl}_{3}\right): \delta_{\mathrm{P}} 24.82 .{ }^{1} \mathrm{H}$ NMR $\left(300.1 \mathrm{MHz}, \mathrm{CDCl}_{3}\right): \delta_{\mathrm{H}} 1.38\left(6 \mathrm{H}, \mathrm{t},{ }^{3} J_{\mathrm{HH}}=7.1\right.$ $\left.\mathrm{Hz}, \mathrm{CH}_{2} \mathrm{CH}_{3}\right), 2.45-2.55\left(2 \mathrm{H}, \mathrm{m}, \mathrm{PCH}_{2}\right), 4.03\left(3 \mathrm{H}, \mathrm{s}, \mathrm{OCH}_{3}\right), 4.08\left(3 \mathrm{H}, \mathrm{s}, \mathrm{OCH}_{3}\right), 4.18(2 \mathrm{H}, \mathrm{dq}$, $\left.{ }^{3} J_{\mathrm{HH}}=7.1 \mathrm{~Hz},{ }^{3} J_{\mathrm{PH}}=7.6 \mathrm{~Hz}, \mathrm{OCH}_{2}\right), 4.89-4.94\left(2 \mathrm{H}, \mathrm{m}, \mathrm{NCH}_{2}\right) .{ }^{13} \mathrm{C} \mathrm{NMR}\left(75.5 \mathrm{MHz}, \mathrm{CDCl}_{3}\right)$ : $\delta_{\mathrm{c}} 16.03\left(\mathrm{~d},{ }^{3} J_{\mathrm{PC}}=6.3 \mathrm{~Hz}, \mathrm{CH}_{3}\right), 26.48\left(\mathrm{~d},{ }^{1} J_{\mathrm{PC}}=140.8 \mathrm{~Hz}, \mathrm{PCH}_{2}\right), 44.77\left(\mathrm{NCH}_{2}\right), 52.30$ $\left(\mathrm{OCH}_{3}\right), 53.13\left(\mathrm{OCH}_{3}\right), 61.80\left(\mathrm{OCH}_{2}\right), 61.88\left(\mathrm{OCH}_{2}\right), 129.48(\mathrm{C}=), 139.78(\mathrm{C}=), 158.30(\mathrm{C}=\mathrm{O})$, $160.10(\mathrm{C}=\mathrm{O})$. Anal. Calcd for $\mathrm{C}_{12} \mathrm{H}_{20} \mathrm{~N}_{3} \mathrm{O}_{7} \mathrm{P} \bullet 0.25 \mathrm{H}_{2} \mathrm{O}: \mathrm{C}, 40.74 ; \mathrm{H}, 5.84 ; \mathrm{N}, 11.88 ; \mathrm{P}, 8.76 \%$. Found: C, 40.37; H, 5.56; N, 11.55; P, 8.44\%.

\section{Dimethyl 1-[3-(diethoxyphosphoryl)propyl]-1H-1,2,3-triazole-4,5-dicarboxylate} (3b). Yellow oil, yield 95\%; IR (thin layer, $\left.v_{\max }, \mathrm{cm}^{-1}\right)$ : 1029, 1060 (P-O-C), $1280(\mathrm{P}=\mathrm{O}), 1736(\mathrm{C}=\mathrm{O})$. ${ }^{31} \mathrm{P}$ NMR $\left(121.5 \mathrm{MHz}, \mathrm{CDCl}_{3}\right): \delta_{\mathrm{P}} 29.71 .{ }^{1} \mathrm{H}$ NMR $\left(300.1 \mathrm{MHz}, \mathrm{CDCl}_{3}\right): \delta_{\mathrm{H}} 1.37\left(6 \mathrm{H}, \mathrm{t},{ }^{3} J_{\mathrm{HH}}=\right.$ 
7.1 Hz, $\left.\mathrm{CH}_{3}\right), 1.76-1.87\left(2 \mathrm{H}, \mathrm{m}, \mathrm{PCH}_{2} \mathrm{CH}_{2}\right), 2.24-2.34\left(2 \mathrm{H}, \mathrm{m}, \mathrm{PCH}_{2}\right), 4.03\left(3 \mathrm{H}, \mathrm{s}, \mathrm{OCH}_{3}\right), 4.06$ $\left(3 \mathrm{H}, \mathrm{s}, \mathrm{OCH}_{3}\right), 4.12-4.18\left(4 \mathrm{H}, \mathrm{m}, \mathrm{OCH}_{2}\right), 4.75\left(2 \mathrm{H}, \mathrm{t},{ }^{3} J_{\mathrm{HH}}=7.1 \mathrm{~Hz}, \mathrm{NCH}_{2}\right) .{ }^{13} \mathrm{C} \mathrm{NMR}(75.5$ $\left.\mathrm{MHz}, \mathrm{CDCl}_{3}\right): \delta_{\mathrm{c}} 16.14\left(\mathrm{~d},{ }^{3} J_{\mathrm{PC}}=6.0 \mathrm{~Hz}, \mathrm{CH}_{3}\right), 22.27\left(\mathrm{~d},{ }^{1} J_{\mathrm{PC}}=139.7 \mathrm{~Hz}, \mathrm{PCH}_{2}\right), 23.25$ $\left(\mathrm{PCH}_{2} \mathrm{CH}_{2}\right), 50.20\left(\mathrm{~d},{ }^{3} J_{\mathrm{PC}}=17.0, \mathrm{NCH}_{2}\right), 52.37\left(\mathrm{OCH}_{3}\right), 53.22\left(\mathrm{OCH}_{3}\right), 61.46\left(\mathrm{OCH}_{2}\right), 61.54$ $\left(\mathrm{OCH}_{2}\right), 129.67(\mathrm{C}=), 139.70(\mathrm{C}=), 158.50(\mathrm{C}=\mathrm{O}), 160.18(\mathrm{C}=\mathrm{O})$. Anal. Calcd for $\mathrm{C}_{13} \mathrm{H}_{22} \mathrm{~N}_{3} \mathrm{O}_{7} \mathrm{P}$ : C, 42.98; H, 6.10; N, 11.57; P, 8.53\%. Found: C, 43.00; H, 6.17; N, 11.43; P, 8.61\%.

Tetramethyl \{1-[2-(diethoxyphosphoryl)ethyl]-1H-1,2,3-triazole-4,5-diyl $\}$ bis(phosphonate) (3c) was prepared by the same procedure from azidophosphonate 1a and tetramethyl acetylenediphosphonate $\mathbf{2 b}$ at $60{ }^{\circ} \mathrm{C}$ for $2 \mathrm{~h}$. Brown oil, yield 95\%; IR (thin layer, $v_{\max }, \mathrm{cm}^{-1}$ ): 1032 (br, P-O-C), 1266 (br, P=O). ${ }^{31} \mathrm{P}$ NMR (121.5 MHz, $\left.\mathrm{CDCl}_{3}\right): \delta$ Р 3.67, 7.45, 24.62. ${ }^{1} \mathrm{H} \mathrm{NMR}$ $\left(300.1 \mathrm{MHz}, \mathrm{CDCl}_{3}\right): \delta_{\text {н }} 1.39\left(6 \mathrm{H}, \mathrm{t},{ }^{3} \mathrm{~J}_{\mathrm{HH}}=7.1 \mathrm{~Hz}, \mathrm{CH}_{3}\right), 2.16-2.25\left(2 \mathrm{H}, \mathrm{m}, \mathrm{PCH}_{2}\right), 3.95(6 \mathrm{H}, \mathrm{s}$, $\left.\mathrm{OCH}_{3}\right), 3.99\left(6 \mathrm{H}, \mathrm{s}, \mathrm{OCH}_{3}\right), 4.17-4.22\left(4 \mathrm{H}, \mathrm{m}, \mathrm{OCH}_{2}\right), 5.00-5.08\left(2 \mathrm{H}, \mathrm{m}, \mathrm{NCH}_{2}\right) .{ }^{13} \mathrm{C} \mathrm{NMR}$ $\left(75.5 \mathrm{MHz}, \mathrm{CDCl}_{3}\right): \delta_{\mathrm{c}} 15.45\left(\mathrm{CH}_{3}\right), 26.57\left(\mathrm{~d},{ }^{1} J_{\mathrm{PC}}=139.9 \mathrm{~Hz}, \mathrm{PCH}_{2}\right), 44.82\left(\mathrm{NCH}_{2}\right), 52.89$ $\left(\mathrm{OCH}_{3}\right), 52.97\left(\mathrm{OCH}_{3}\right), 53.36\left(\mathrm{OCH}_{3}\right), 53.43\left(\mathrm{OCH}_{3}\right), 61.17\left(\mathrm{OCH}_{2}\right), 61.20\left(\mathrm{OCH}_{2}\right), 129.35(\mathrm{dd}$, $\left.{ }^{1} J_{\mathrm{PC}}=216.4 \mathrm{~Hz},{ }^{2} J_{\mathrm{PC}}=34.1 \mathrm{~Hz}, \mathrm{PC}=\right), 141.56\left(\mathrm{dd},{ }^{1} J_{\mathrm{PC}}=236.8 \mathrm{~Hz},{ }^{2} J_{\mathrm{PC}}=20.3 \mathrm{~Hz}, \mathrm{PC}=\right)$. Anal. Calcd for $\mathrm{C}_{12} \mathrm{H}_{26} \mathrm{~N}_{3} \mathrm{O}_{9} \mathrm{P}_{3}:$ C, 32.08; H, 5.83; N, 9.35\%. Found: C, 32.00; H, 5.91; P, 9.30\%.

Tetramethyl 2H-1,2,3-triazole-4,5-diylbis(phosphonate) (5). Tetramethyl acetylenediphosphonate $\mathbf{2 b}(400 \mathrm{mg}, 1.65 \mathrm{mmol})$. was slowly dropped to the solution of sodium azide $(113 \mathrm{mg}, 1.73 \mathrm{mmol})$ in water $(5 \mathrm{ml})$ at room temperature. The reaction mixture was stirred for $0.5 \mathrm{~h}$, followed by the acidification to $\mathrm{pH}=3$ by aq. $\mathrm{HCl}$. Then water was removed under reduced pressure, the residue was dissolved in acetonitrile $(6 \mathrm{ml}), \mathrm{NaCl}$ precipitated was filtered off, and filtrate was concentrated in vacuo and dried over $\mathrm{P}_{2} \mathrm{O}_{5}$ to give the final white solid, yield $81 \%$, $380 \mathrm{mg}, \mathrm{mp} 100{ }^{\circ} \mathrm{C}$; IR (KBr, $\left.v_{\max }, \mathrm{cm}^{-1}\right)$ : 1029 (P-O-C), 1246 (P=O). ${ }^{31} \mathrm{P}$ NMR (121.5 MHz, $\left.\mathrm{CDCl}_{3}\right): \delta$ Р 8.49. ${ }^{1} \mathrm{H}$ NMR $\left(300.1 \mathrm{MHz}, \mathrm{CDCl}_{3}\right): \delta_{\text {н }} 3.90(12 \mathrm{H}, \mathrm{s}, \mathrm{OCH} 3), 13.87(1 \mathrm{H}, \mathrm{br} . \mathrm{s}, \mathrm{NH})$. ${ }^{13} \mathrm{C}$ NMR $\left(75.5 \mathrm{MHz}, \mathrm{CDCl}_{3}\right): \delta_{\mathrm{c}} 54.05\left(\mathrm{OCH}_{3}\right), 54.12\left(\mathrm{OCH}_{3}\right), 128.59\left(\mathrm{dd},{ }^{1} J_{\mathrm{PC}}=237.0 \mathrm{~Hz}\right.$, $\left.{ }^{3} J_{\mathrm{PC}}=27.0 \mathrm{~Hz}, \mathrm{C}-\mathrm{C}\right)$. Anal. Calcd for $\mathrm{C}_{6} \mathrm{H}_{13} \mathrm{~N}_{3} \mathrm{O}_{6} \mathrm{P}_{2} \bullet 0.25 \mathrm{NaCl}: \mathrm{C}, 24.28 ; \mathrm{H}, 4.41 ; \mathrm{N}, 14.16 \%$. Found: C, 24.19; H, 4.50; N, 14.36\%.

Diethyl [2-[4,5-bis(amino)carbonyl]-1H-1,2,3-triazol-1-yl]alkyl]phosphonates (6a-c,7) (general procedure). The solution of the corresponding triazole 3a,b (2.0 mmol) and amine (4.0 $\mathrm{mmol})$ in methanol $(5 \mathrm{ml})$ was vigorously stirred at room temperature until the completion of the reaction according ${ }^{31} \mathrm{P}$ NMR and TLC monitoring of the reaction course (namely, 12, 14 and 24 $\mathrm{h}$ for $\mathbf{6 b}, \mathbf{6 c}$, and $\mathbf{6 a}$, respectively). Then solvent was removed under reduced pressure to give products with high purity.

Diethyl [3-[4,5-bis[(butylamino)carbonyl]-1H-1,2,3-triazol-1-yl]propyl]phosphonate (6a). Brown oil, 91\%; IR (thin layer, $\left.v_{\max }, \mathrm{cm}^{-1}\right)$ : 1030, $1058(\mathrm{P}-\mathrm{O}-\mathrm{C}), 1245(\mathrm{P}=\mathrm{O}), 1676(\mathrm{C}=\mathrm{O}) .{ }^{31} \mathrm{P}$ NMR (121.5 MHz, $\left.\mathrm{CDCl}_{3}\right): \delta$ Р 30.37. ${ }^{1} \mathrm{H}$ NMR (300.1 MHz, $\left.\mathrm{CDCl}_{3}\right): \delta_{\text {н }} 0.88-0.94\left(6 \mathrm{H}, \mathrm{m}, \mathrm{CH}_{3}\right.$ in $\left.{ }^{\mathrm{n}} \mathrm{Bu}\right), 1.26\left(6 \mathrm{H}, \mathrm{t},{ }^{3} \mathrm{~J}_{\mathrm{HH}}=7.1 \mathrm{~Hz}, \mathrm{CH}_{3}\right), 1.34-1.42\left(4 \mathrm{H}, \mathrm{m}, \underline{\mathrm{CH}_{2}} \mathrm{CH}_{3}\right), 1.56-1.61(4 \mathrm{H}, \mathrm{m}$, $\left.\underline{\mathrm{CH}_{2}} \mathrm{CH}_{2} \mathrm{CH}_{3}\right), 1.67-1.79\left(2 \mathrm{H}, \mathrm{m}, \mathrm{PCH}_{2} \underline{\mathrm{CH}}_{2}\right), 2.11-2.23\left(2 \mathrm{H}, \mathrm{m}, \mathrm{PCH}_{2}\right), 3.32-3.46(4 \mathrm{H}, \mathrm{m}$, $\left.\mathrm{NHCH}_{2}\right), 4.02-4.07\left(4 \mathrm{H}, \mathrm{m}, \mathrm{OCH}_{2}\right), 4.95\left(2 \mathrm{H}, \mathrm{t},{ }^{3} J_{\mathrm{HH}}=6.9 \mathrm{~Hz}, \mathrm{NCH}_{2}\right), 7.78(1 \mathrm{H}$, br. s, NH), $11.13\left(1 \mathrm{H}\right.$, br. s, NH). ${ }^{13} \mathrm{C}$ NMR $\left(75.5 \mathrm{MHz}, \mathrm{CDCl}_{3}\right): \delta \mathrm{c} 15.24\left(\mathrm{CH}_{3}\right.$ in $\left.\mathrm{Bu}\right), 16.74\left(\mathrm{~d},{ }^{3} J_{\mathrm{PC}}=6.0\right.$ 
$\left.\mathrm{Hz}, \mathrm{CH}_{3}\right), 21.88\left(\mathrm{CH}_{2}\right), 26.31\left(\mathrm{CH}_{2}\right), 27.44\left(\mathrm{~d},{ }^{1} J_{\mathrm{PC}}=138.3 \mathrm{~Hz}, \mathrm{PCH}_{2}\right), 29.29\left(\mathrm{CH}_{2}\right), 31.06$ $\left(\mathrm{CH}_{2}\right), 48.83\left(\mathrm{NCH}_{2}\right), 62.39\left(\mathrm{OCH}_{2}\right), 62.46\left(\mathrm{OCH}_{2}\right), 131.45(\mathrm{C}=), 139.32(\mathrm{C}=), 157.08(\mathrm{C}=\mathrm{O})$, $161.72(\mathrm{C}=\mathrm{O})$. Anal. Calcd for $\mathrm{C}_{19} \mathrm{H}_{36} \mathrm{~N}_{5} \mathrm{O} 5 \mathrm{P}: \mathrm{C}, 51.22 ; \mathrm{H}, 8.15 ; \mathrm{P}, 6.95 \%$. Found: $\mathrm{C}, 50.93 ; \mathrm{H}$, $8.01 ; \mathrm{P}, 6.53 \%$.

Diethyl [2-[4,5-bis[[2-(1-pyrrolidinyl)ethyl]amino]-1H-1,2,3-triazol-1-yl]ethyl]phosphonate (6b). Brown oil, 98\%; IR (thin layer, $\left.v_{\max }, \mathrm{cm}^{-1}\right)$ : 1025, 1056 (P-O-C), $1251(\mathrm{P}=\mathrm{O}), 1676(\mathrm{C}=\mathrm{O})$. ${ }^{31} \mathrm{P}$ NMR (121.5 MHz, $\left.\mathrm{CDCl}_{3}\right): \delta \mathrm{P} 26.96 .{ }^{1} \mathrm{H} \mathrm{NMR}\left(300.1 \mathrm{MHz}, \mathrm{CDCl}_{3}\right): \delta \mathrm{H} 1.35\left(6 \mathrm{H}, \mathrm{t},{ }^{3} J_{\mathrm{HH}}=\right.$ $\left.7.0 \mathrm{~Hz}, \mathrm{CH}_{3}\right), 1.78-1.79\left(8 \mathrm{H}, \mathrm{m},-\mathrm{CH}_{2}\left(\mathrm{CH}_{2}\right)_{2} \mathrm{CH}_{2}\right), 2.54-2.59\left(8 \mathrm{H}+2 \mathrm{H}, \mathrm{m},-\mathrm{CH}_{2}\left(\mathrm{CH}_{2}\right)_{2} \mathrm{CH}_{2}-\right.$ and $\left.\mathrm{PCH}_{2}\right), 2.71\left(2 \mathrm{H}, \mathrm{t},{ }^{3} J_{\mathrm{HH}}=6.0 \mathrm{~Hz}, \mathrm{NHCH}_{2} \underline{\mathrm{CH}}_{2} \mathrm{~N}\right), 2.73\left(2 \mathrm{H}, \mathrm{t},{ }^{3} J_{\mathrm{HH}}=6.0 \mathrm{~Hz}, \mathrm{NHCH}_{2} \underline{\mathrm{CH}}_{2} \mathrm{~N}\right)$, $3.56\left(2 \mathrm{H}, \mathrm{t},{ }^{3} J_{\mathrm{HH}}=6.0 \mathrm{~Hz}, \mathrm{NHCH}_{2}\right), 3.58\left(2 \mathrm{H}, \mathrm{t},{ }^{3} J_{\mathrm{HH}}=6.0 \mathrm{~Hz}, \mathrm{NHCH}_{2}\right), 4.19\left(4 \mathrm{H}, \mathrm{dq},{ }^{3} J_{\mathrm{PH}}=\right.$ $\left.8.0 \mathrm{~Hz},{ }^{3} \mathrm{~J}_{\mathrm{HH}}=7.0 \mathrm{~Hz}, \mathrm{OCH}_{2}\right), 5.06-5.09\left(2 \mathrm{H}, \mathrm{m}, \mathrm{NCH}_{2} \mathrm{CH}_{2} \mathrm{P}\right) .{ }^{13} \mathrm{C} \mathrm{NMR}\left(75.5 \mathrm{MHz}, \mathrm{CDCl}_{3}\right): \delta_{\mathrm{C}}$ $16.81\left(\mathrm{~d},{ }^{3} J_{\mathrm{PC}}=6.0 \mathrm{~Hz}, \mathrm{CH}_{3}\right), 23.95\left(-\mathrm{CH}_{2}\left(\mathrm{CH}_{2}\right)_{2} \mathrm{CH}_{2^{-}}\right), 27.49\left(\mathrm{~d},{ }^{1} J_{\mathrm{PC}}=138.5 \mathrm{~Hz}, \mathrm{PCH}_{2}\right), 38.84$

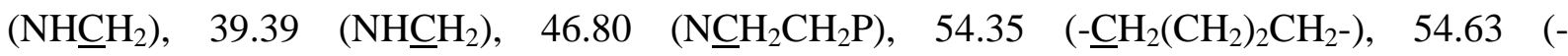
$\left.\mathrm{CH}_{2}\left(\mathrm{CH}_{2}\right)_{2} \mathrm{CH}_{2}\right), 55.28\left(\mathrm{NHCH}_{2} \underline{\mathrm{CH}}_{2} \mathrm{~N}\right), 62.39\left(\mathrm{OCH}_{2}\right), 62.51\left(\mathrm{OCH}_{2}\right), 131.43(\mathrm{C}=), 139.32$ $(\mathrm{C}=), 157.09(\mathrm{C}=\mathrm{O}), 161.76(\mathrm{C}=\mathrm{O})$. Anal. Calcd for $\mathrm{C}_{22} \mathrm{H}_{40} \mathrm{~N}_{7} \mathrm{O}_{5} \mathrm{P}: \mathrm{C}, 51.45 ; \mathrm{H}, 7.85 ; \mathrm{P}, 6.03 \%$. Found: C, 51.21; H, 7.91; N, 6.30\%.

Diethyl [2-[4,5-bis[[[2-(2-pyridinyl)ethyl]amino]carbonyl]-1H-1,2,3-triazol-1-yl]ethyl]phosphonate (6c). Brown oil, 97\%; IR (thin layer, $\left.v_{\max }, \mathrm{cm}^{-1}\right)$ : 1026, 1054 (P-O-C), $1253(\mathrm{P}=\mathrm{O})$, $1676(\mathrm{C}=\mathrm{O}) .{ }^{31} \mathrm{P} \mathrm{NMR}\left(121.5 \mathrm{MHz}, \mathrm{CDCl}_{3}\right): \delta_{\mathrm{P}} 26.46 .{ }^{1} \mathrm{H} \mathrm{NMR}\left(300.1 \mathrm{MHz}, \mathrm{CDCl}_{3}\right): \delta_{\text {н }} 1.33$ $\left(6 \mathrm{H}, \mathrm{t},{ }^{3} J_{\mathrm{HH}}=7.0 \mathrm{~Hz}, \mathrm{CH}_{3}\right), 2.44\left(2 \mathrm{H}, \mathrm{dt},{ }^{2} J_{\mathrm{PH}}=18.0 \mathrm{~Hz},{ }^{3} J_{\mathrm{HH}}=8.0 \mathrm{~Hz}, \mathrm{PCH}_{2}\right), 2.73\left(2 \mathrm{H}, \mathrm{t},{ }^{3} J_{\mathrm{HH}}\right.$ $\left.=6.0 \mathrm{~Hz}, \mathrm{NHCH}_{2} \underline{\mathrm{CH}}_{2} \mathrm{~N}\right), 3.56\left(2 \mathrm{H}+2 \mathrm{H}, \mathrm{t},{ }^{3} J_{\mathrm{HH}}=6.0 \mathrm{~Hz}, \mathrm{NHCH}_{2} \underline{\mathrm{CH}}_{2} \mathrm{~N}\right.$ and $\left.\mathrm{NHCH}_{2}\right), 3.86(2 \mathrm{H}$, $\left.\mathrm{t},{ }^{3} J_{\mathrm{HH}}=6.0 \mathrm{~Hz}, 2 \mathrm{H}, \mathrm{NHCH}_{2}\right), 4.14\left(4 \mathrm{H}, \mathrm{dq},{ }^{3} J_{\mathrm{PH}}=8.0 \mathrm{~Hz},{ }^{3} J_{\mathrm{HH}}=7.0 \mathrm{~Hz}, \mathrm{OCH}_{2}\right), 5.10(2 \mathrm{H}, \mathrm{dt}$, $\left.{ }^{3} J_{\mathrm{PH}}=8.0 \mathrm{~Hz},{ }^{3} J_{\mathrm{HH}}=8.0 \mathrm{~Hz}, \mathrm{NCH}_{2} \mathrm{CH}_{2} \mathrm{P}\right), 7.14-7.24,7.56-7.63,8.55-8.61(4 \mathrm{H}+2 \mathrm{H}+2 \mathrm{H}$, all m, $\left.\mathrm{C}_{5} \mathrm{H}_{4} \mathrm{~N}\right) .{ }^{13} \mathrm{C}$ NMR $\left(75.5 \mathrm{MHz}, \mathrm{CDCl}_{3}\right): \delta_{\mathrm{c}} 16.82\left(\mathrm{~d},{ }^{3} J_{\mathrm{PC}}=6.0 \mathrm{~Hz}, \mathrm{CH}_{3}\right), 27.46\left(\mathrm{~d},{ }^{1} J_{\mathrm{PC}}=138.5\right.$ $\left.\mathrm{Hz}, \mathrm{PCH}_{2}\right), 37.17\left(\underline{\mathrm{CH}}_{2}-\mathrm{C}_{5} \mathrm{H}_{4} \mathrm{~N}\right), 38.06\left(\underline{\mathrm{CH}}_{2}-\mathrm{C}_{5} \mathrm{H}_{4} \mathrm{~N}\right), 39.13\left(\mathrm{NH}_{\underline{C}} \mathrm{H}_{2}\right), 39.80\left(\mathrm{NHCH}_{2}\right), 46.80$ $\left(\mathrm{NCH}_{2} \mathrm{CH}_{2} \mathrm{P}\right), 62.40\left(\mathrm{OCH}_{2}\right), 62.52\left(\mathrm{OCH}_{2}\right), 121.91\left(\mathrm{C}^{5}\right), 122.14\left(\mathrm{C}^{5}\right), 123.74\left(\mathrm{C}^{3}\right), 131.33(\mathrm{C}=)$, $136.76\left(\mathrm{C}^{4}\right), 137.03\left(\mathrm{C}^{4}\right), 139.31(\mathrm{C}=), 149.87\left(\mathrm{C}^{6}\right), 157.08(\mathrm{C}=\mathrm{O}), 159.20\left(\mathrm{C}^{2}\right), 161.62(\mathrm{C}=\mathrm{O})$. Anal. Calcd for $\mathrm{C}_{24} \mathrm{H}_{32} \mathrm{~N}_{7} \mathrm{O}_{5} \mathrm{P}: \mathrm{C}, 54.44 ; \mathrm{H}, 6.09 ; \mathrm{P}, 5.85 \%$. Found: C, 54.15; H, 5.91; P, $5.69 \%$.

Diethyl [2-(4,5,6,7,8,9-hexahydro-4,9-dioxo-1H-1,2,3-triazolo[4,5-f][1,4]diazocin-1-yl)ethyl]phosphonate (7) was prepared by the same procedure from 1 eq. of ethylenediamine $(2 \mathrm{mmol})$ in methanol (30 ml) after 7 days. Off-white hygroscopic powder, 95\%, mp 64-65 ${ }^{\circ} \mathrm{C}$; $\mathrm{IR}(\mathrm{KBr}$, $\left.v_{\max }, \mathrm{cm}^{-1}\right):$ 1024, $1055(\mathrm{P}-\mathrm{O}-\mathrm{C}), 1253(\mathrm{P}=\mathrm{O}), 1647,1676(\mathrm{C}=\mathrm{O}) .{ }^{31} \mathrm{P}$ NMR $(121.5 \mathrm{MHz}$, $\left.\mathrm{CDCl}_{3}\right): \delta_{\mathrm{P}} 25.85 .{ }^{1} \mathrm{H}$ NMR $\left(300.1 \mathrm{MHz}, \mathrm{CDCl}_{3}\right): \delta_{\mathrm{H}} 1.38\left(6 \mathrm{H}\right.$, br. s, $\left.\mathrm{CH}_{3}\right), 2.47-2.53(2 \mathrm{H}, \mathrm{m}$, $\left.\mathrm{PCH}_{2}\right), 3.52-3.72\left(4 \mathrm{H}, \mathrm{m}, \mathrm{NHCH}_{2} \underline{\mathrm{CH}}_{2} \mathrm{NH}\right), 4.17-4.19\left(4 \mathrm{H}, \mathrm{m}, \mathrm{OCH}_{2}\right), 5.14\left(2 \mathrm{H}\right.$, br. s, $\left.\mathrm{NCH}_{2}\right)$. ${ }^{13} \mathrm{C}$ NMR $\left(75.5 \mathrm{MHz}, \mathrm{CDCl}_{3}\right): \delta_{\mathrm{C}} 16.81\left(\mathrm{~d},{ }^{3} J_{\mathrm{PC}}=6.0 \mathrm{~Hz}, \mathrm{CH}_{3}\right), 27.43\left(\mathrm{~d},{ }^{1} J_{\mathrm{PC}}=138.5 \mathrm{~Hz}\right.$, $\left.\mathrm{PCH}_{2}\right), 46.80\left(\mathrm{NCH}_{2}\right), 48.85\left(\mathrm{NCH}_{2} \underline{\mathrm{CH}}_{2} \mathrm{~N}\right), 62.40\left(\mathrm{OCH}_{2}\right), 62.51\left(\mathrm{OCH}_{2}\right), 131.35(\mathrm{C}=), 139.30$ $(\mathrm{C}=), 157.08(\mathrm{C}=\mathrm{O}), 161.63(\mathrm{C}=\mathrm{O})$. Anal. Calcd for $\mathrm{C}_{12} \mathrm{H}_{20} \mathrm{~N}_{5} \mathrm{O}_{5} \mathrm{P} \bullet 0.5 \mathrm{H}_{2} \mathrm{O}: \mathrm{C}, 40.68 ; \mathrm{H}, 5.94 ; \mathrm{N}$, 19.77; P, 8.74\%. Found: C, 40.71; H, 5.64; N, 19.23; P, 8.56\%.

\section{Diethylammonium 1-[3-(diethoxyphosphoryl)propyl]-5-(methoxycarbonyl)-1H-1,2,3-} triazole-4-carboxylate (8). The solution of the triazole $\mathbf{3 b}(130 \mathrm{mg}, 0.4 \mathrm{mmol})$ and diethylamine 
$(62 \mathrm{mg}, 0.82 \mathrm{mmol})$ in methanol $(2 \mathrm{ml})$ was vigorously stirred at room temperature for 30 days. Then solvent was removed under reduced pressure and the residue was purified by column chromatography on silica gel (chloroform-ethanol=10:1) to afford the salt $\mathbf{8}$ as a yellowish oil, 95 $\mathrm{mg}, 56 \%$; IR (thin layer, $\left.v_{\max }, \mathrm{cm}^{-1}\right)$ : 1028, $1060(\mathrm{P}-\mathrm{O}-\mathrm{C}), 1231(\mathrm{P}=\mathrm{O}), 1640\left(\mathrm{C}=\mathrm{O}\right.$ in $\left.\mathrm{COO}^{-}\right)$, $1728\left(\mathrm{C}=\mathrm{O}\right.$ in COOMe). ${ }^{31} \mathrm{P} \mathrm{NMR}\left(121.5 \mathrm{MHz}, \mathrm{CDCl}_{3}\right): \delta$ P 30.35. ${ }^{1} \mathrm{H}$ NMR $(300.1 \mathrm{MHz}$, $\left.\mathrm{CDCl}_{3}\right): \delta_{\mathrm{H}} 1.26\left(6 \mathrm{H}, \mathrm{t},{ }^{3} J_{\mathrm{HH}}=7.0 \mathrm{~Hz}, \mathrm{OCH}_{2} \underline{\mathrm{CH}}_{3}\right), 1.33\left(6 \mathrm{H}, \mathrm{t},{ }^{3} J_{\mathrm{HH}}=7.3 \mathrm{~Hz}, \mathrm{CH}_{3}\right), 1.69-1.78$ $\left(2 \mathrm{H}, \mathrm{m}, \mathrm{PCH}_{2} \mathrm{CH}_{2}\right), 2.15-2.26\left(2 \mathrm{H}, \mathrm{m}, \mathrm{PCH}_{2}\right), 3.06\left(4 \mathrm{H}, \mathrm{q},{ }^{3} \mathrm{~J}_{\mathrm{HH}}=7.3 \mathrm{~Hz}, \mathrm{CH}_{2}\right), 3.88(3 \mathrm{H}, \mathrm{s}$, $\left.\mathrm{OCH}_{3}\right), 3.98-4.08\left(4 \mathrm{H}, \mathrm{m}, \mathrm{OCH}_{2}\right), 4.64\left(2 \mathrm{H}, \mathrm{t},{ }^{3} J_{\mathrm{HH}}=6.9 \mathrm{~Hz}, \mathrm{NCH}_{2}\right), 9.45\left(2 \mathrm{H}, \mathrm{br} . \mathrm{s}, \mathrm{NH}_{2}\right)$. Free acid was obtained as an oil $(53 \mathrm{mg})$ after acidification by $5 \%$ aq. $\mathrm{HCl}$. folowed by extraction with chloroform, drying over $\mathrm{Na}_{2} \mathrm{SO}_{4}$ and concentration in vacuo. ${ }^{31} \mathrm{P} \mathrm{NMR}\left(121.5 \mathrm{MHz}, \mathrm{CDCl}_{3}\right): \delta_{\mathrm{P}}$ 29.80. ${ }^{1} \mathrm{H} \mathrm{NMR}\left(300.1 \mathrm{MHz}, \mathrm{CDCl}_{3}\right): \delta_{\mathrm{H}} 1.29\left(6 \mathrm{H}, \mathrm{t},{ }^{3} \mathrm{~J}_{\mathrm{HH}}=7.0 \mathrm{~Hz}, \mathrm{OCH}_{2} \underline{\mathrm{CH}}_{3}\right), 1.73-1.82(2 \mathrm{H}$, $\left.\mathrm{m}, \mathrm{PCH}_{2} \underline{\mathrm{CH}}_{2}\right), 2.18-2.28\left(2 \mathrm{H}, \mathrm{m}, \mathrm{PCH}_{2}\right), 4.02-4.10\left(4 \mathrm{H}, \mathrm{m}, \mathrm{OCH}_{2}\right), 4.11\left(3 \mathrm{H}, \mathrm{s}, \mathrm{OCH}_{3}\right), 4.90$ $\left(2 \mathrm{H}, \mathrm{t},{ }^{3} \mathrm{~J}_{\mathrm{HH}}=7.1 \mathrm{~Hz}, \mathrm{NCH}_{2}\right)$.

\section{Acknowledgements}

This work was supported by the Program of Chemistry and Material Science Division of the RAS and the program of President of Russian Federation "For Young PhD scientists" (No MK425.2010.3). The authors express their deep gratitude for the gift of tetramethyl acetylenediphosphonate to Dr. A.V. Dogadina and Prof. B.I. Ionin (Saint-Petersburg State Institute of Technology).

\section{References}

1. (a) Kolb, H.; Sharpless, K. B. Drug Discovery Today, 2003, 24, 1128. (b) Kolb, H. C.; Finn, M. G.; Sharpless, K. B. Angew. Chem. Int. Ed. 2001, 40, 2004.

2. Huisgen, R. in 1,3-Dipolar Cycloaddition Chemistry; Padwa, A. Ed.; Wiley: New York, 1984.

3. For reviews see, e.g., (a) Meldal, M.; Tornøe, C. W. Chem. Rev. 2008, 108, 2952. (b) Tron, G. C.; Pirali, T.; Billington, R. A.; Canonico, P. L.; Sorba, G.; Genazzani, A. A. Med. Res. Rev. 2008, 28, 278. (c) Mamat, C. Mini-Rev. Org. Chem. 2009, 6, 21. (d) Struthers, H.; Mindt, T. L.; Schibliet, R. Dalton Trans. 2010, 39, 675.

4. Chittaboina, S.; Xie, F.; Wang, Q. Tetrahedron Lett. 2005, 46, 2331.

5. (a) Kuijpers, B. H. M.; Groothuys, S.; Keereweer, A. R.; Quaedflieg, P. J. L. M.; Blaauw, R. H.; van Delft, F. L.; Rutjes, F. P. J. T. Org. Lett. 2004, 6, 3123. (b) Dondoni, A.; Giovannini, P. P.; Massi, A. Org. Lett. 2004, 6, 2929.

6. Weller, R. L.; Rajski, S. R. Org. Lett. 2005, 7, 2141.

7. Al-Masoudi, N. A.; Al-Soud, Y. A.; Abdul-Zahra, A. Heteroatom Chem. 2004, 15, 380. 
8. (a) Alvarez, R.; Velazquez, S.; San-Felix, A.; Aquaro, S.; Clercq, E. D.; Perno, C. F.; Karlesson, A.; Balzarini, J.; Camarasa, M. J. J. Med. Chem. 1994, 37, 4185. (b) Vela'zquez, S.; Alvarez, R.; Perez, C.; Gago, F.; DeClercq, E.; Alzarini, J.; Camarasa, M. J. Antiviral Chem. Chemother. 1998, 9, 481.

9. Palhagen, S.; Canger, R.; Henriksen, O.; vanParys, J. A.; Riviere, M.-E.; Karolchyk, M. A. Epilepsy Res. 2001, 43, 115.

10. (a) Genin, M. J.; Allwine, D. A.; Anderson, D. J.; Barbachyn, M. R.; Emmert, D. E.; Garmon, S. A.; Graber, D. R.; Greda, K. C.; Hester, J. B.; Hutchinson, D. K.; Morris, J.; Reischer, R. J.; Ford, C. W.; Zurenco, G. E.; Hamel, J. C.; Schaadt, R. D.; Stapert, D.; Yagi, B. H. J. Med. Chem. 2000, 43, 953. (b) Reck, F.; Zhou, F.; Girardot, M.; Kern, G.; Eyermann, C. J.; Hales, N. J.; Ramsay, R. R.; Gravestock, M. B. J. Med. Chem. 2005, 48, 499.

11. Krise, J. P.; Stella, V. J. Adv. Drug Delivery Rev. 1996, 19, 287.

12. Skarpos, H.; Osipov, S. N.; Vorob'eva, D. V.; Odinets, I.L.; Lork, E.; Röschenthaler, G.-V. J. Org. Biomol. Chem. 2007, 5, 2361.

13. Artyushin, O. I; Osipov, S. N.; Röschenthaler, G.-V.; Odinets, I. L. Synthesis 2009, 21, 3579.

14. Artyushin, O. I.; Vorob'eva, D. V.; Vasil'eva, T. P.; Osipov, S. N.; Röschenthaler, G.-V.; Odinets, I. L. Heteroatom Chem. 2008, 19, 293.

15. Vorobyeva, D. V.; Karimova, N. M.; Vasilyeva, T. P.; Osipov, S. N.; Odinets, I. L.; Röschenthaler, G.-V. J. Fluor. Chem. 2010, 131, 378.

16. Coats, S. J.; Link, J. S.; Gauthier, D.; Hlasta, D. J. Org. Lett. 2005, 7,1469.

17. Chre'tien, F.; Gross, B. J. Heterocycl. Chem. 1982, 19, 263.

18. Périon, R.; Ferrières, V.; García-Moreno, M. I.; Ortiz Mellet, C.; Duval, R.; García Fernández, J. M.; Plusquellec D. Tetrahedron 2005, 61, 9118.

19. Mayot, E.; Gérardin-Charbonnier, C.; Selve, C. J. Fluor. Chem. 2005, 126, 715.

20. Yap, A. H.; Weinreb, S. M. Tetrahedron Lett. 2006, 47, 3035.

21. (a) Grieco, P. A. Organic Synthesis in Water; Blackie Academic and Professional: London, 1998. (b) Organic Reactions in Water: Principles, Strategies and Applications; Lindström, U. M. Ed.; Blackwell: Oxford, 2007. (c) Li, C.-J.; Chan, T.-H. Comprehensive Organic Reactions in Aqueous Media; Wiley \& Sons: New York, 2007. (d) Paradowska, J.; Stodulski, M.; Mlynarski, J. Angew. Chem. Int. Ed. 2009, 48, 4288. (e) Butler, R. N.; Coyne A. G. Chem. Rev. 2010, 110, 6302.

22. Narayan, S.; Muldoon, J.; Finn, M. G.; Fokin, V. V.; Kolb, H. C.; Sharpless, K. B. Angew. Chem. Int. Ed. 2005, 44, 3275.

23. (a) Wang, Z.-X.; Qin, H.-L. Chem. Commun. 2003, 2450. (b) Driez-Gonzralez, S.; Correa, A.; Cavallo, L.; Nolan, S. P. Chem.-Eur. J. 2006, 12, 7558. (c) Driez-Gonzralez, S.; Nolan, S. P. Angew. Chem., Int. Ed., 2008, 47, 8881. (d) Driez-Gonzralez, S.; Stevens E. D.; Nolan, S. P. Chem. Commun. 2008, 4747. (e) Ozçubukçu, S.; Ozkal, E.; Jimeno, C.; Perica`s, M. A. Org. Lett. 2009, 11, 4680. (f) Candelon, N.; Lastrerou`eres, D.; Diallo, A. K.; Ruiz Aranzanes, J.; Astruc, D.; Vincent, J.-M. Chem. Commun. 2008, 741. 
24. (a) Garcia-Alvarez, J.; Diez, J.; Gimeno, J. Green Chem. 2010, 12, 2127. (b) Youcef, R. A.; Dos Santos, M.; Roussel, S.; Lubin-Germain, N.; Uziel, J.; Baltaze, J.-P. J. Org. Chem. 2009, 74, 4318. (c) Gonzaga, F.; Yu, G.; Brook, M. A. Chem. Commun. 2009, 13, 1730.

25. (a) Kumar, I.; Rode, C. V. Chem. Lett. 2007, 35, 592. (b) Kumar, I.; Rode, C. V.; Rana, S.; Cho, J. W. Tetrahedron: Asymmetry 2010, 21, 352.

26. Semakin, A. N.; Sukhorukov, A. Yu.; Lesiv, A. V.; Khomutova, Yu. A.; Ioffe, S. L. Russ. J. Org. Chem. 2007, 43, 1218.

27. Matveeva, E.V.; Odinets, I. L. Current Organic Chem. 2010, 14, 1171.

28. Ramasamy, K.; Tam, R.; Averett, D. US Patent 6130326, 2000.

29. (a) Rheingold, A. L.; Liable-Sands, L. M.; Trofimenko, S. Angew. Chem. Int. Ed. 2000, 39, 3321. (b) Rheingold, A. L.; Liable-Sands, L. M.; Trofimenko, S. Inorg. Chim. Acta 2002, $330,38$.

30. Dolomanov, O. V.; Bourhis, L. J. Gildea, R. J.; Howard, J. A. K.; Puschmann, H. J. Appl. Cryst. 2009, 42, 339.

31. Sheldrick, G. Acta Cryst. Sect. A 2008, 64, 112. 